\title{
Segmental quantitative analysis of digital thallium-201 myocardial scintigrams in diagnosis of coronary artery disease Comparison with rest and exercise electrocardiography and
coronary arteriography
}

\author{
R J WAINWRIGHT, M N MAISEY, E SOWTON \\ From the Department of Nuclear Medicine and the Department of Cardiology, Guy's Hospital, London
}

SUMMARY One hundred and forty-nine patients with suspected ischaemic heart disease were evaluated by exercise thallium-201 myocardial scintigraphy $\left({ }^{201} \mathrm{Tl}\right.$ SMS), single lead exercise electrocardiography, and coronary arteriography. Myocardial distribution of tracer was assessed semi-quantitatively from digital ${ }^{201} \mathrm{Tl}$ scintigrams and compared with tracer distribution in subjects with normal hearts. Fifty-two of $54(96 \%)$ patients with normal coronary arteries had normal myocardial scintigrams whereas three patients had a positive ischaemic exercise electrocardiogram and were scan normal. Conversely, 36 of 95 (38\%) patients with coronary artery disease had a positive ischaemic electrocardiogram compared with 94 of $95(99 \%)$ patients who had a positive myocardial scintigram. Disease in specific coronary arteries could be deduced from the topography of myocardial tracer deficit. Disease was predicted correctly in 76 out of $80(95 \%)$ of left anterior descending coronary stenoses, in 48 out of $64(75 \%)$ of right coronary artery stenoses, and in 55 out of $64(85 \%)$ of left circumflex coronary artery stenoses, despite the presence of infarcted myocardium in other territories. Similarly, single vessel disease was predicted correctly in 14 out of $17(82 \%)$ patients and multiple vessel disease in 64 out of $77(83 \%)$ patients. ${ }^{201} \mathrm{Tl}$ SMS with segmental quantitative analysis is a highly sensitive and specific technique in the diagnosis of coronary artery disease and may be a useful screening procedure to select patients for further investigation, particularly those with evidence of life-threatening severe left coronary artery disease.

Stress myocardial imaging using thallium-201 $\left({ }^{201} \mathrm{Tl}\right.$ SMS) has challenged the predictive accuracy of conventional non-invasive procedures in the detection of patients with ischaemic heart disease. Indeed, Bailey et al. ${ }^{1}$ suggested that this technique was more sensitive than 12 lead exercise electrocardiography. Several studies have since claimed that ${ }^{201} \mathrm{Tl}$ SMS is: more sensitive than single lead exercise electrocardiography and completely specific ${ }^{2}$; more sensitive than single lead exercise electrocardiography but equally specific $^{3}$; of similar sensitivity to 12 lead electrocardiography but probably more specific ${ }^{4}$; less sensitive than exercise electrocardiography but certainly more specific. ${ }^{5}$ Other studies have completely neglected this comparison. ${ }^{6}$ There is general agreement that the diagnostic sensitivity of Received for publication 10 March 1981 exercise electrocardiography in the detection of patients with ischaemic heart disease is enhanced by ${ }^{201} \mathrm{Tl}$ SMS, the combined test sensitivity being greate than $90 \%$ in many series.

These disparate results cloud the individual contribution of ${ }^{201} \mathrm{Tl}$ myocardial imaging to the screening and evaluation of patients with ischaemic heart disease. Undoubtedly one of the major causes of confusion is the use of imprecise and unstandardised definitions of tracer deficit found within the ${ }^{201} \mathrm{Tl}$ myocardial scintigram which may appear in several different forms, for example as an analogue image on Polaroid film, as a radiographic transparency, or as a computer processed digital image displayed in monotone or colour on a television monitor. Previous definitions of significant tracer deficit have included: focal count reduction (unspecified degree) occupying 
at least $15 \%$ of the left ventricular circumference ${ }^{1}$; focal reduction in activity greater than $50 \%$ (unspecified area, judged qualitatively on Polaroid film ${ }^{3}$; focal deficit of five qualitative categories ${ }^{4}$; focal activity less than $75 \%$ of the isocount contour level ${ }^{6}$; focal area of 'total or near total absence of activity'; focal deficit 'equal to or less than lung activity'. Occasionally no criteria for the evaluation of abnormal images are stated. ${ }^{9}$ These imprecise definitions are frequently accompanied by insufficient discussion of the variable scintigraphic anatomy of coronary artery disease.

Following an analysis of the topography of ${ }^{201} \mathrm{Tl}$ defects produced by lesions in specific coronary arteries, ${ }^{10}$ this study was undertaken to compare the diagnostic sensitivity and specificity of ${ }^{201} \mathrm{Tl}$ SMS in comparison to single lead (V5) exercise electrocardiography in the detection and prediction of patients with single vessel and multiple vessel coronary artery disease using an objective and highly reproducible analysis of digital ${ }^{201} \mathrm{Tl}$ myocardial scintigrams.

\section{Patients and methods}

One hundred and forty-nine patients (118 men and 31 women; mean age 50 years; range 24 to 67 years) with suspected ischaemic heart disease were evaluated by coronary arteriography and ${ }^{201} \mathrm{Tl}$ SMS according to the availability of radionuclide. All patients complained of chest pain as their predominant limiting symptom. Full physical examination, resting 12 lead electrocardiogram, chest $x$-ray film, and biochemical screen were performed as part of a routine assessment. Patients with recent myocardial infarction (within three months), unstable angina pectoris, previous cardiac surgery, valvular or congenital heart disease, and hypertension were excluded.

Cardioactive drugs, particularly beta-blockers, were withdrawn from most patients before investigation. In all cases myocardial imaging and coronary arteriography were performed during the same hospital admission of approximately five days.

Among 95 patients with arteriographically proven coronary artery disease 44 patients gave a typical history of previous myocardial infarction; 37 patients had a persistent pathological $Q$ wave or loss of $r$ wave in the electrocardiogram; seven patients had no electrocardiographic evidence of previous myocardial infarction. In contrast three patients gave no history of myocardial infarction but did have a pathological $Q$ wave or loss of $r$ wave in the resting electrocardiogram and were therefore included in the myocardial infarction group.

Among the 48 patients without evidence of previous myocardial infarction 28 had a completely normal resting electrocardiogram, 13 had isolated abnormalities of the $T$ wave, six had ST segment depression at rest, and one had complete left bundlebranch block.

\section{CORONARY ARTERIOGRAPHY}

Selective coronary arteriography and left ventriculography were performed using the Sones or Judkins technique. Exposures were made on $35 \mathrm{~mm}$ cine-film at 50 frames/s using a 6" 'image intensifier'. Selective coronary arteriograms were obtained in standard left anterior oblique and right anterior oblique projections after injection of 6 to $8 \mathrm{ml}$ of urografin into each coronary artery. Left ventriculography was performed in the right anterior oblique projection using 40 to $50 \mathrm{ml}$ urografin. All arteriograms were considered technically adequate and reported by three observers without knowledge of the scintigraphic findings. Note was made of the site and severity of stenoses and the presence of coronary collateral vessels. Stenoses were expressed as percentage reductions in lumen diameter and considered haemodynamically significant if $50 \%$ or more. Left ventricular function was assessed visually from the contrast ventriculogram.

\section{EXERCISE TESTING}

After an initial rest electrocardiogram all patients performed a maximal exercise test in the upright position on a Monark bicycle ergometer with monitoring of a single V5 praecordial electrode until the onset of limiting symptoms such as angina pectoris or undue breathlessness and fatigue. An exercise electrocardiogram was regarded as positive for ischaemia if $1 \mathrm{~mm}$ or more ST segment depression was seen $0.08 \mathrm{~s}$ after the $\mathrm{J}$ point in three consecutive beats. ${ }^{201} \mathrm{Tl}, 2 \mathrm{mCi}$, was injected through an indwelling intravenous cannula at the final exercise level which was then maintained for approximately one and a half to two minutes.

\section{MYOCARDIAL SCINTIGRAPHY}

Myocardial imaging was started 10 minutes after termination of the exercise test using an Ohio-Nuclear Series 100 scintillation camera and a high sensitivity parallel hole collimator. A persistence oscilloscope was used to position the patient carefully beneath the scintillation carmera ensuring that the myocardial image appeared in the central field of view. The heart was surrounded with a layer of lead cape placed over the praecordium to reduce the contribution of counts from regions other than the myocardium. Three views (anterior, left anterior oblique $45^{\circ}$ and $55^{\circ}$ ) were acquired with the patient supine, rotating the camera head around the chest in each view. Finally a full left lateral projection was performed with the patient on 
his right side. 200000 counts were acquired in each projection using a $20 \%$ window centred about the $72.5 \mathrm{keV}$ mercury $x$-ray peak emitted in $98 \%$ abundance from ${ }^{201} \mathrm{Tl}$ decay. Each image took approximately six minutes to collect and was simultaneously recorded on radiographic transparency film (Kodak NMB 179) and magnetic disc after digital conversion to a $64 \times 64$ matrix using a dedicated minicomputer (DEC Gamma 11).

\section{INTERPRETATION OF MYOCARDIAL IMAGES}

The digital images of each patient were enhanced by a $50 \%$ background erase, interpolated and displayed in 16 grades of colour on a television monitor. Each colour grade was linearly related to tracer activity and corresponded approximately to a $3 \%$ change in count rate in the final image. By comparison with stored data of myocardial ${ }^{201} \mathrm{Tl}$ profiles obtained from 22 patients with a normal heart it was possible to define image abnormalities objectively in cases of suspected ischaemic heart disease.

Myocardial segments falling below our established 95\% confidence limits were regarded as significant areas of tracer deficit. These regions corresponded approximately to a 12 to $15 \%$ change in count rate (four colour grades) over an area approximately 14\% of the myocardial circumference. All scintigrams were interpreted without knowledge of the corresponding coronary anatomy. Prediction of lesions in individual coronary arteries was based on knowledge of the scintigraphic anatomy of coronary artery disease described in the preceding paper. Statistical comparison of the data was performed using the $\chi^{2}$ test.

\section{Results}

Fifty-four patients had normal coronary arteries, 18 patients had single vessel disease, 38 patients had double vessel disease, 33 patients had triple vessel disease, and six patients had quadruple vessel disease. There was a significant stenosis in the left anterior descending coronary artery in 80 patients, in the right coronary artery in 64 patients and in the left circumflex coronary artery in 65 patients.

Three of the 54 patients with normal coronary arteries had a positive exercise test and a normal myocardial scintigram. These patients were considered to have false positive electrocardiographic stress tests. In comparison 52 out of 54 patients had a completely normal scintigram (specificity $96 \%$ ). One patient with false positive tracer deficit had complete left bundle-branch block; the other patient with apparently normal coronary arteries had tracer deficit in the distribution of the right coronary artery. This patient was later shown to have a reproducible spasm of the right coronary artery during a second coronary arteriogram.

In the 95 patients with coronary artery disease the conventional single lead exercise electrocardiogram $\frac{\bar{\sigma}}{\omega}$ was positive for ischaemia in only 36 of $95(38 \%) \overrightarrow{\widetilde{d}}$ patients while 94 of $95(99 \%)$ patients had abnormal myocardial scintigrams $(p<0.0005)$. If chest pain on $\%$ exertion was included as a positive ischaemic response $\overrightarrow{0}$ then still only 57 out of $95(60 \%)$ patients with $\vec{\overrightarrow{ }}$ coronary artery disease were identified by exercise $\vec{\omega}$ testing alone. The inclusion of patients with an abnormal $\mathrm{Q}$ wave or loss of $\mathrm{r}$ wave, however, produced a total diagnostic yield of 74 out of $95(78 \%)$ के patients using conventional methods of detection, though this proportion still remained significantly less $\infty$ than that achieved by scintigraphy.

(a) PATIENTS WITHOUT MYOCARDIAL

INFARCTION

The comparative results of exercise electrocardiography and ${ }^{201} \mathrm{Tl}$ SMS in the 48 patients with no evidence of previous myocardial infarction are shown in Fig. 1. The rest electrocardiogram was entirely normal in $28(58 \%)$ patients and became :abnormal on exercise in only eight patients. In contrast 27 of these patients developed significant exercise-induced scintigraphic abnormalities which correctly predicted single vessel disease in six patients and multiple vessel disease in 16 patients (Table 1). Prediction of single or multiple vessel disease was not possible from analysis of the electrocardiographic data alone but this might have been improved by use of a multiple lead electrocardiograph recording system. Among 19 patients with resting abnormalities of the $T$

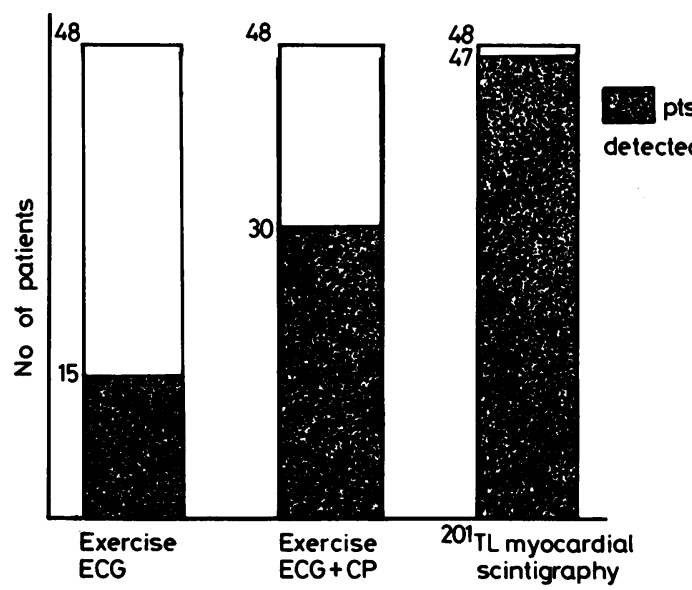

Fig. 1 Comparison of single lead exercise electrocardiography and exercise ${ }^{201} \mathrm{Tl}$ myocardial scintigraphy in 48 patients with no evidence of myocardial infarction. CP, cardiac pain. 
Table 1 Extent of coronary artery disease predicted by ${ }^{201} \mathrm{Tl}$ myocardial scintigraphy in patients without myocardial infarction

\begin{tabular}{lccccc}
\hline \multicolumn{5}{c}{ Scintigraphic prediction } \\
\hline & No. of & & & & \\
& Trteries diseased & 0 & 1 & 2 & 3 \\
Angiographic disease & 1 & 1 & 6 & 0 & 0 \\
& 2 & 0 & 4 & 7 & 0 \\
& 3 & 0 & 1 & 3 & 6 \\
\hline
\end{tabular}

wave or ST segment, only seven patients developed a positive ischaemic response during exercise testing whereas the myocardial scintigram became abnormal in all patients. One patient with complete left bundle-branch block preventing interpretation of the exercise electrocardiogram had evidence of myocardial ischaemia in the scintigram.

Thus, a total of 47 of 48 (98\%) patients with coronary artery disease and non-infarcted myocardium were correctly detected by myocardial scintigraphy compared with only 15 of $48(31 \%)$ patients who were detected by single lead exercise electrocardiography $(p<0.0005)$. If exertional chest pain was included as a positive response for ischaemia then still significantly fewer patients $(62 \%)$ were correctly detected by an exercise test alone.

(b) PATIENTS WITH MYOCARDIAL INFARCTION The comparative results of exercise electrocardiography and ${ }^{201} \mathrm{Tl}$ SMS in the 47 patients with historical or electrocardiographic evidence of previous myocardial infarction are shown in Fig. 2. There were 26 anterior myocardial infarctions, 18 inferior

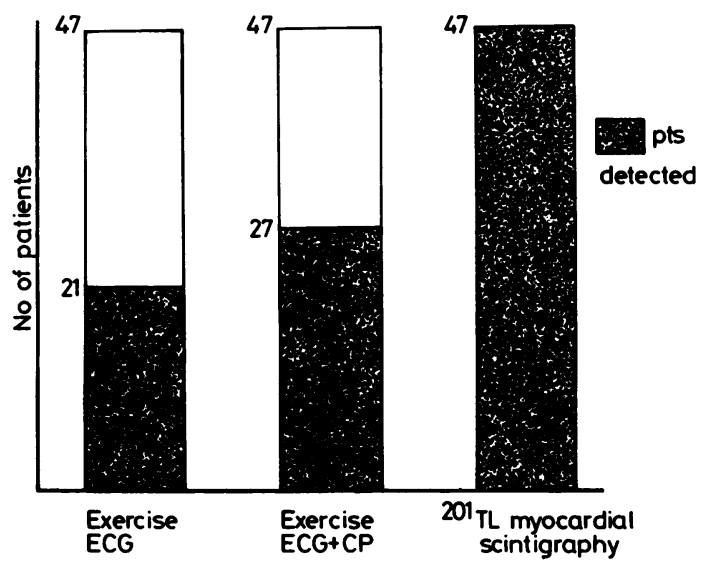

Fig. 2 Comparison of single lead exercise electrocardiography and exercise ${ }^{201} \mathrm{Tl}$ myocardial scintigraphy in 47 patients with evidence of previous myocardial infarction. CP, cardiac pain. myocardial infarctions, and three lateral myocardial infarctions. A total of 47 occluded vessels were found in these patients, a number significantly higher than in the group without myocardial infarction. Fortythree of these occluded vessels were associated with abnormal tracer deficit compared with 16 of 19 occlusions causing positive tracer deficit in patients without myocardial infarction (NS). Though triple vessel disease was significantly more prevalent in these patients than in those without myocardial infarction, there was no difference in the workload achieved at the end of exercise in either group.

All 47 patients had an abnormal exercise myocardial scintigram whereas only 40 patients ( $85 \%$ ) had a pathological $Q$ wave or loss of $r$ wave in the rest electrocardiogram and only 21 patients (45\%) developed a positive ischaemic electrocardiogram response during exercise. Twenty-seven patients $(57 \%)$ had both chest pain and a positive ischaemic electrocardiographic response.

DETECTION OF INDIVIDUAL CORONARY ARTERY STENOSES

The site of a pathological $Q$ wave or loss of $r$ wave in the resting electrocardiogram usually correlated with severe disease in a corresponding coronary artery. Most patients with anterior myocardial infarction had complete occlusion of the left anterior descending coronary artery and all patients with inferior myocardial infarction had complete occlusion of the right coronary artery or a dominant left circumflex artery.

In patients without myocardial infarction it was not possible to detect accurately lesions in individual coronary arteries from analysis of the resting electrocardiogram or single lead exercise electrocardiographic response alone. It was assumed that the lead V5 vector would detect left anterior descending coronary disease optimally and indeed of the 15 patients with a positive ischaemic electrocardiographic response 13 had disease in the left anterior descending coronary artery, but the majority of these patients also had disease in other coronary vessels.

In comparison Table 2 shows the results of ${ }^{201} \mathrm{Tl}$ SMS in the detection of specific coronary artery stenoses in patients with ischaemic and infarcted myocardium. Similar numbers of diseased coronary arteries were detected in each group.

Most false negative scintigrams for left anterior descending coronary disease occurred in patients who had moderate stenosis (50 to $60 \%$ ) of the left anterior descending artery and concomitant severe double or triple vessel disease which itself produced appropriate regional tracer deficit. One patient had considerable hypertrophy of the first septal artery which probably facilitated preservation of myocardial perfusion in the 
Table 2 Detection sensitivity of ${ }^{201} \mathrm{Tl}$ SMS for specific coronary artery disease subtending viable and necrotic myocardium

\begin{tabular}{lll}
\hline $\begin{array}{l}\text { Coronary artery } \\
\text { diseased }\end{array}$ & $\begin{array}{l}\text { Non-infarcted } \\
\text { myocardium }\end{array}$ & $\begin{array}{l}\text { Infarcted } \\
\text { myocardium }\end{array}$ \\
\hline $\begin{array}{l}\text { Left anterior } \\
\text { descending }\end{array}$ & $40 / 42(95 \%)$ & $36 / 38(95 \%)$ \\
$\begin{array}{l}\text { Left circumflex } \\
\text { Right coronary }\end{array}$ & $24 / 29(83 \%)$ & $31 / 36(86 \%)$ \\
\hline
\end{tabular}

left anterior descending coronary artery territory. In the group without myocardial infarction left anterior descending coronary artery disease was not associated with false positive scintigrams, but three false positive appearances did occur in the group with myocardial infarction. One of these patients had sustained a previous anterior myocardial infarction with normal appearances of the coronary arteries at the time of angiography. Presumably a coronary embolus or prolonged coronary artery spasm accounted for myocardial infarction in this patient.

False negative scintigrams for left circumflex coronary artery disease occurred in patients with stenoses of only moderate severity (50 to $70 \%$ ) or in patients with extensive triple vessel disease. Only one scintigram was falsely positive for left circumflex coronary disease.

False negative scintigrams for right coronary artery disease were more common, occurring in 16 patients; eight were associated with well-developed retrograde collateral filling of the right coronary artery which may have been responsible for preventing relative myocardial ischaemia ${ }^{11}$; seven had only moderate right coronary artery disease (50 to $60 \%$ stenosis) often associated with severe disease in other coronary arteries; one had disease in a recessive right coronary artery which presumably did not subtend sufficient myocardium to produce a scintigraphic abnormality. One patient had single vessel right coronary artery disease ( $50 \%$ stenosis in the atrioventricular groove) which was not detected by myocardial scintigraphy or exercise electrocardiography. This patient was the only subject in whom both noninvasive investigations were falsely negative. One false positive scintigram for right coronary artery disease resulted from extension of severe septal tracer deficit into the adjacent inferior myocardial wall in a patient with a large left ventricular aneurysm.

Significant disease in specific coronary arteries was correctly predicted in a total of 76 of $80(95 \%)$ left anterior descending coronary stenoses, 55 of $65(85 \%)$ left circumflex coronary stenoses, and 48 of 64 (75\%) right coronary artery stenoses. Single vessel disease was corrctly predicted in 14 of $17(82 \%)$ patients and multiple vessel disease in 64 of $77(83 \%)$ patients.

DETECTION OF DISEASE IN NONINFARCTED MYOCARDIUM

As previously mentioned patients with previous myocardial infarction could usually be identified by an abnormal rest electrocardiogram, but this feature was unhelpful in the prediction of disease in coronary arteries other than those associated with infarcted myocardium.

The sensitivity of ${ }^{201} \mathrm{Tl}$ SMS in detecting coronary artery disease subtending noninfarcted myocardium in the presence of regional myocardial necrosis is shown in Fig. 3. In 29 patients with anterior and anterolateral myocardial infarction, all of whom had tracer deficit in the distribution of the left anterior descending coronary artery, there were 39 potential areas of myocardial ischaemia caused by disease in the remaining arteries, $28(72 \%)$ of these potential anginaproducing regions were detected by ${ }^{201} \mathrm{Tl}$ SMS. Only one patient had a false negative scintigram in the distribution of both remaining arteries supplying noninfarcted myocardium.

Similarly in 18 patients with inferior myocardial infarction, 16 of whom had associated inferior wall tracer deficit, there were 26 regions of noninfarcted myocardium subtended by significantly diseased coronary arteries; $24(92 \%)$ of these potential regions of ischaemia were detected by myocardial scintigraphy. No patient had a false negative scintigram in the left anterior descending and left circumflex coronary artery territories when right coronary artery occlusion was responsible for myocardial infarction; only one patient had false negative tracer deficit in the territory of the left anterior descending coronary artery. This region was subtended by a $60 \%$ stenosis.

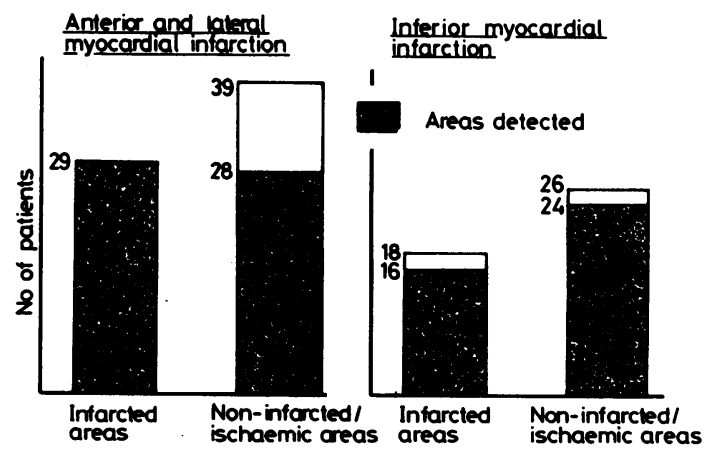

Fig. 3 Sensitivity of exercise ${ }^{201} \mathrm{Tl}$ myocardial scintigraphy in detection of coronary artery disease subtending noninfarcted myocardium when infarcted myocardium was also present. 
Altogether 45 out of 47 regions of myocardial infarction were detected by ${ }^{201} \mathrm{Tl}$ SMS in comparison with 52 out of 65 noninfarcted but potentially ischaemic zones $(p<0.05)$, indicating that regional necrosis is scintigraphically more apparent than regional ischaemia. Nevertheless, there was no significant difference in the diagnostic sensitivity of ${ }^{201} \mathrm{Tl}$ SMS in the detection of patients with or without previous myocardial infarction as shown in Fig. 1 and 2.

\section{Discussion}

The introduction of ${ }^{201} \mathrm{Tl}$ as a myocardial imaging agent in $\operatorname{man}^{12}$ has added a new dimension to the noninvasive evaluation of patients with coronary heart disease. During the stress of dynamic exercise, regions of myocardium subtended by significant coronary stenoses accumulate relatively less administered tracer than normal myocardium and thus appear as 'cold spots' in scintigrams acquired immediately after exercise. The investigator using this technique is perhaps fortunate that the majority of significant stenoses are situated in the proximal coronary arteries and therefore subtend large segments of left ventricular myocardium. These regions correspondingly appear as extensive areas of tracer depletion in the myocardial scintigram. Despite the current lack of rigour in the definition of a 'cold spot' it is claimed that both the diagnostic sensitivity and specificity of conventional stress electrocardiography has been enhanced by simultaneous ${ }^{201} \mathrm{Tl}$ SMS. ${ }^{3} 4$

The results of this study differ in several important respects from previously reported work comparing the diagnostic efficiency of ${ }^{201}$ Tl SMS and exercise electrocardiography. Firstly, scintigraphic analysis is performed after digital conversion and simple enhancement of the image. Scintigrams which are displayed on Polaroid film or radiographic transparency are marred by high background activity of tracer and limited dynamic range of the film. Variations of image intensity may cause problems in interpretation and only gross deficit of tracer accumulation can be identified unequivocally. Secondly, focal deficit of tracer is carefully defined in a semiquantitative manner by reference to the known distribution of myocardial tracer accumulation found in normal subjects. Defects are defined on the basis of 95\% confidence limits established for tracer activity in different myocardial segments. The analysis is precise and takes into account the large variation of tracer activity found within the normal myocardial profile. Moreover vague definitions of tracer deficit such as 'areas of inhomogeneity in the scan'2 or definitions which entirely beg the question, viz. a 'definite area of decreased uptake ${ }^{5}$ are avoided. Using this method of scintigraphic analysis the sensitivity of ${ }^{201} \mathrm{Tl}$ myocardial imaging in the detection of patients with coronary artery disease in this series is very high (99\%); only one patient, who had single vessel right coronary artery disease, was undetected by ${ }^{201} \mathrm{Tl}$ SMS. This high sensitivity was not achieved by sacrificing specificity; most patients with normal coronary arteries had normal myocardial scintigrams. Some of these patients gave a convincing history of angina pectoris and even presented as acute coronary insufficiency; many were thought to have sustained previous myocardial infarction. The arteriographic evidence of normal coronary arteries combined with a normal exercise ${ }^{201} \mathrm{Tl}$ myocardial scintigram, however, supports the hypothesis that chest pain in the majority of these subjects has a non-ischaemic basis.

Although not every diseased coronary artery was associated with tracer deficit it was encouraging to find that most patients $(83 \%)$ with multiple vessel disease could be correctly identified by ${ }^{201} \mathrm{Tl}$ SMS. Similarly, the majority of patients with single vessel disease $(82 \%)$ were also correctly identified and not overdiagnosed as patients with multiple vessel disease.

The presence of infarcted myocardium did not appear to distort the detection of coronary disease in arteries subtending noninfarcted myocardium. For example, left anterior descending coronary artery disease was detected by scintigraphy in 10 out of 11 patients with previous inferior myocardial infarction. The only false negative scintigram occurred in a patient with a $60 \%$ proximal left anterior descending coronary stenosis which was treated without venous bypass graft surgery. Despite the absence of symptoms several young patients with previous inferior myocardial infarction are currently being investigated by coronary arteriography in order to detect residual life-threatening stenoses in coronary arteries supplying viable myocardium. In the absence of severe disease in the left coronary artery many of these patients can be strongly reassured; others may need urgent referral for coronary artery surgery. Our results indicate that ${ }^{201} \mathrm{Tl}$ SMS alone may be sufficiently sensitive to screen patients with previous inferior myocardial infarction and select for coronary arteriography those patients with a high probability of severe left coronary artery disease.

It has been suggested by Turner et al. ${ }^{4}$ that patients with typical angina pectoris are not suitable candidates for screening with ${ }^{201} \mathrm{Tl}$ SMS because the post-test probability of coronary artery disease is still high even when a negative test result is obtained. Thus, in their series of patients investigated by ${ }^{201} \mathrm{Tl}$ SMS, given a negative test result the post-test probability of disease was still high $(86 \%)$ when the 
pre-test disease prevalence was high (95\%). Their test sensitivity, however, was only $68 \%$, probably because observers were reporting images from Polaroid film rather than from digital scintigrams improved by simple image processing and quantification. This study shows that ${ }^{201} \mathrm{TI}$ SMS can be made sufficiently sensitive to reduce the post-test probability of disease to $8 \%$ given a negative test result when the prevalence of disease is very high $(\mathbf{9 0 \%})$, for example in patients with typical angina pectoris. The index of clinical suspicion for coronary artery disease in this group of patients, however, is so high that the addition of a less than perfect test alters the post-test probability of disease relatively little. Therefore, it seems appropriate to investigate most patients with typical angina pectoris by performing immediate coronary arteriography.

It can be shown that the largest difference between pre-test and post-test probabilities of disease are found when disease prevalence lies between 16 and $50 \%$, for example in the group of patients with atypical angina pectoris and non-anginal chest pain. ${ }^{13}$ Moreover, the probability of disease given a negative test result is very small indeed, such that most patients could forego diagnostic coronary arteriography. This policy reduces the number of patients exposed to the risk and discomfort of unnecessary cardiac catheterisation. Botvinick et al. ${ }^{14}$ using rubidium-81 have also suggested that patients with atypical angina pectoris are the most suitable group to investigate with stress myocardial perfusion imaging.

The detection of ${ }^{201} \mathrm{Tl}$ myocardial perfusion deficit in asymptomatic patients with coronary artery disease raises a clinical dilemma for which there is yet no satisfactory solution as there are no reliable data to suggest that particular medical or surgical regimens will significantly influence morbidity and mortality in this subpopulation..$^{15}$ Moreover, it may be necessary to settle the question of false positive tracer deficit by performing definitive coronary arteriography quite frequently in order to avoid erroneous labelling of normal patients with a diagnosis of coronary artery disease.

The quantitative approach to ${ }^{201} \mathrm{Tl}$ SMS described in this study is designed to hold the diagnostic criterion level for reporting defects at a constant optimum. Further improvements in predictive accuracy, however, can be attained by adopting a Bayesian strategy ${ }^{16}$ of altering the criterion level of the test in accordance with varying prior probabilities of disease estimated from the clinical setting, as shown by Swensson et al. ${ }^{17}$ who showed that the availability of clinical information at the time of reporting improved the diagnostic accuracy of radiograms.

In conclusion, ${ }^{201} \mathrm{Tl}$ SMS is a highly sensitive and specific technique for the detection and localisation of established coronary artery disease. It may be used to provide an objective assessment of the extent of myocardial ischaemia independent from the coronary arteriogram and can differentiate ischaemic from necrotic tissue, ${ }^{18}$ thus identifying angina-producing regions of the myocardium. The measurement of ${ }^{201} \mathrm{Tl}$ myocardial uptake, however, is relative, not absolute; defects are identified by comparison with the most normal region of the scintigram. Moreover the most severe coronary stenosis may produce early angina pectoris and limit exercise before detectable tracer depletion has occurred in regions subtended by less critical coronary lesions. Both reasons preclude the detection of all coronary disease in every patient entirely from analysis of the patterns of myocardial tracer deficit. Nevertheless this study indicates that the majority of important coronary artery lesions, particularly left anterior descending coronary stenoses, cause clear abnormalities of tracer accumulation which allow important and accurate decisions on further patient management.

Dr R J Wainwright was supported by a British Heart Foundation grant. This work has been submitted as part of a London MD Thesis.

\section{References}

1 Bailey IK, Griffith LSC, Rouleau J, Strauss HW, Pitt B. Thallium-201 myocardial perfusion imaging at rest and during exercise. Comparative sensitivity to electrocardiography in coronary artery disease. Circulation 1977; 55: 79-87.

2 Carillo AP, Marks DS, Pickard SD, Khaja F, Goldstein S. Correlation of exercise ${ }^{201}$ thallium myocardial scan with coronary arteriograms and the maximal exercise test. Chest 1978; 73: 321-6.

3 Ritchie JL, Zaret BL, Strauss HW, et al. Myocardial imaging with thallium-201; a multicentre study in patients with angina pectoris or acute myocardial infarction. Am ₹ Cardiol 1978; 42: 345-50.

4 Turner DA, Battle WE, Deshmukh $\mathrm{H}$, et al. The predictive value of myocardial perfusion scintigraphy after stress in patients without previous myocardial infarction. F Nucl Med 1978; 19: 249-55.

5 Verani MS, Marcus ML, Razzak MA, Ehrhardt JC. Sensitivity and specificity of thallium-201 perfusion scintigrams under exercise in the diagnosis of coronary artery disease. $\mathrm{F}$ Nucl Med 1978; 19: 773-82.

6 Lenaers A, Block P, Thiel EV, et al. Segmental analysis of T1-201 stress myocardial scintigraphy. $\mathcal{F}$ Nucl Med 1977; 18: 509-16. 
7 Hamilton GW, Trobaugh GB, Ritchie JL, Williams DL, Weaver WD, Gould KL. Myocardial imaging with intravenously injected thallium-201 in patients with suspected coronary artery disease. Analysis of technique and correlation with electrocardiographic, coronary anatomic and ventriculographic findings. Am $\mathcal{f}$ Cardiol 1977; 39: 347-54.

8 Wackers FJT, Lie KI, Liem KL, et al. Potential value of thallium-201 scintigraphy as a means of selecting patients for the coronary care unit. Br Heart $f$ 1979; 41: 111-7.

9 Botvinick EH, Taradash MR, Shames DM, Parmley WW. Thallium-201 myocardial perfusion scintigraphy for the clinical clarification of normal, abnormal and equivocal electrocardiographic stress tests. Am $\mathcal{F}$ Cardiol 1978; 41: 43-51.

10 Wainwright RJ, Maisey MN, Curry PVL, Sowton E. Localisation of coronary artery disease by thallium-201 stress myocardial scintigraphy (abstract). Br Heart $\mathcal{f}$ 1978; 40: 1063.

11 Wainwright RJ, Maisey MN, Edwards AC, Sowton E. Functional significance of coronary collateral circulation during dynamic exercise evaluated by thallium-201 myocardial scintigraphy. Br Heart $\mathcal{f}$ 1980; 43: 47-55.

12 Strauss HW, Harrison K, Langan JK, Lebowtitz E, Pitt B. Thallium-201 for myocardial imaging. Relation of thallium-201 to regional myocardial perfusion. Circulation 1975; 51: 641-5.
13 Diamond GA, Forrester JS. Analysis of probablility as an aid in the clinical diagnosis of coronary-artery disease. $N$ Engl f Med 1979; 300: 1350-8.

14 Botvinick EH, Shames DM, Gershengorn KM, Carlsson E, Ratshin RA, Parmley WW. Myocardial stress perfusion scintigraphy with rubidium-81 versus stress electrocardiography. Am $\mathcal{F}$ Cardiol 1977; 39: 364-71.

15 Wynne J, Cohn LH, Collins JJ, Cohn PF. Myocardial revascularization in patients with multivessel coronary artery disease and minimal angina pectoris. Circulation 1978; 58, suppl 1: I-92-5.

16 Bayes T. An essay towards solving a problem in the doctrine of chances. Philos Trans $R$ Soc 1763; 53: 370.

17 Swensson RG, Hessel RJ, Herman PG. Omissions in radiology: faulty search or stringent reporting criteria Radiology 1977; 123: 563-7.

18 Pohost GM, Zir LM, Moore RH, McKusick KA, Guiney TE, Beller GA. Differentiation of transiently ischaemic from infarcted myocardium by serial imaging after a single dose of thallium-201. Circulation 1977; 55: 294-302.

Requests for reprints to $\mathrm{Dr} R \mathrm{~J}$ Wainwright, Cardiology Department, Guy's Hospital, London SE1 9RT. 